\title{
Two-stage selection strategy in plant breeding
}

\author{
N. W. Simmonds
}

Edinburgh School of Agriculture, West Mains Road, Edinburgh, EH9 3JG, Scotland.

The efficiency of two-stage selection depends upon the correlation of performance between stages. This paper gives a general treatment of two-stage selection on a frequency basis (selected/not selected), taking the costs of trials into account. A table that permits the calculation of the relevant $2 \times 2$ tables from knowledge of $r$ and selection rates is presented. Given that the breeder is prepared to make certain initial subjective judgements/choices, optima, based on numbers of superior selections or least costs usually emerge. If the correlation is not well known it will usually be better to guess low than to guess high. Optimal solutions (which may or may not sharp) may imply the adjustment of total numbers of entries, either upwards or downwards. Examples from rubber and sugarcane breeding are discussed.

\section{INTRODUCTION}

Several authors (Keuls and Sieben, 1955; Gilbert, 1961 ; England, 1967) have pointed out that correlations between successive stages of selection can be used to enhance efficiency but the matter has never been fully explored in terms of explicit strategies. Here I give a treatment of two-stage selection, taking into account the cost of trials. My interest in the matter was generated by rubber breeding data in Malaysia (Ong, 1981) and by discussions on sugarcane breeding problems in the West Indies with Mr D. I. T. Walker. Essentially, the approach is to think of correlation tables as being reduced to $2 \times 2$ frequency-of-selection tables. The converse problem, that of inferring correlations from contingency tables ("tetrachoric correlation"), has been treated by Digby (1983).

\section{TERMINOLOGY}

I assume that the entities before the plant breeder are effectively fixed; that is, they are clones or inbred lines or even outbred lines which have attained sufficient genetic individuality (e.g. grasses-England, 1967). The breeder selects first in a preliminary trial at rate $S_{1}$ and tests the survivors in a more elaborate trial, arriving at a number of "superior" selections from the whole operation. "Superior" is defined as lying in a top fraction $\left(S_{2}\right)$ of the whole population as determined by the results of the second trial. Thus $S_{1}$ is an actual selection rate whereas $S_{2}$ is a notional frequency of superior genotypes. The correlation diagram is resolved into a $2 \times 2$ table (fig. 1), resulting from $N$ total initial entries, of which $(b+d)$ survives the first selection. For actual calculations, the complement of a tetrachoric correlation table is necessary but is not, so far as I know, available. I am much indebted to Dr H. D. Patterson for the calculations summarised in the Appendix. For any $S_{1}, S_{2}$ and $r, b$ can be read and the $2 \times 2$ table calculated, knowing $N$. I assume that the preliminary trials have a unit cost per entry and that the second trial is more expensive, costing $P$ units per entry. For illustration, I take $P$ in the range 5 to 50 . Then the cost of a double cycle of selection is $C=N+P(b+d)$ units.

The breeder cannot hope to retain all the superior selections (unless $r=1$ ) so must choose where to set $S_{1}$ to retain $b$ but reject $a$ (fig. 1); thus his objective is $b$ superior selections. I define $m$ as $b /(a+b)$, the fraction of the superior class which it is hoped to retain. Obviously (fig. 1), if $S_{1}$ is weak, $m$ is large and little of value will be discarded; with small $m$, the mean of the survivors will rise but some of the best are likely to be lost and very small $m$ must be disgenic unless $r$ is very high. I therefore arbitrarily consider $m$ in the range $0 \cdot 2$ to $0 \cdot 8$. 


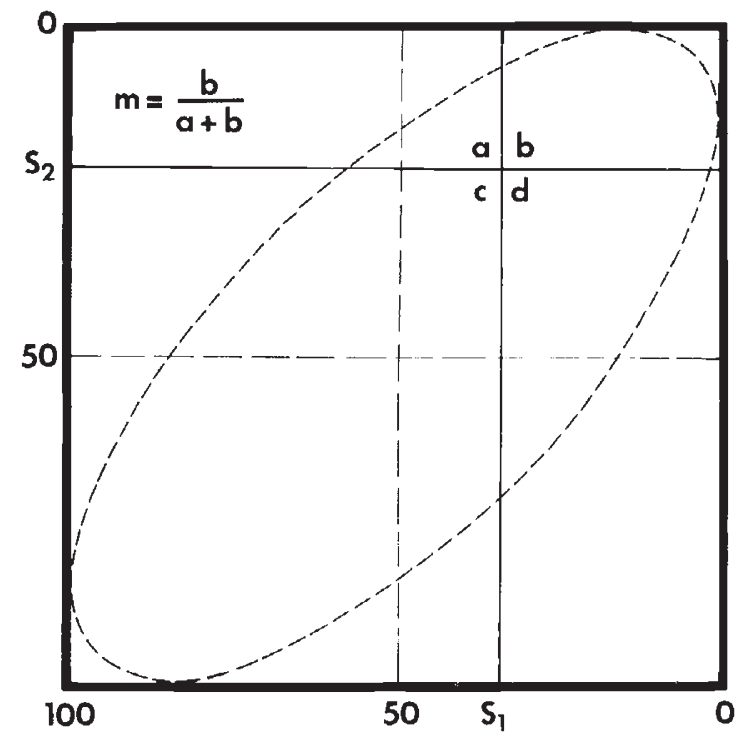

Figure 1 Two-stage selection from correlated trials. The dotted oval line is an outer probability contour of the correlation ellipse. $S_{1}$ is the actual selection rate in the first stage; $S_{2}$ (a notional selection rate) defines the fraction of the population in which the breeder is interested. The correlation ellipse is resolved into a $2 \times 2$ table with four cells of size $a, b, c, d$. The ratio $m$ defines the proportion of the truly superior fraction which is actually retained. The breeder's objective is to maximise $b$ or to minimise the cost of obtaining a defined $b$.

\section{RESULTS}

I assume, first, that the breeder's objective is to preserve into the second stage $b=10$ selections, superior at the level determined by choice of $S_{2}$, that $r$ is known and that neither $N$ nor $C$ is fixed. Then it is possible to calculate the consequences of different choices of $S_{1}$. This is readily done by plotting graphs of $S_{1}$ against $m$ for various $r$ at $S_{2}$ $=5,10,20$ per cent (say). From these graphs, tables such as table 1 can be constructed for any desired
Table 1 Example of calculations for $S_{2}=5$ per cent, $r=0 \cdot 5$ and superior selections $b=10$. The bold figures under $C$ are near-minima (see text). Entries in the column $C$ are nearminima (see text). Entries in the column $(b+d)$ (see figure 1) are the numbers of selections that enter the second-stage trial

\begin{tabular}{rrrrrrr}
\hline & & & & \multicolumn{3}{c}{$C$} \\
\cline { 4 - 7 }$S_{1}$ & $N$ & $m$ & $(b+d)$ & $P=40$ & $P=20$ & $P=5$ \\
\hline 38.5 & 250 & $0 \cdot 80$ & $96 \cdot 3$ & 4102 & 2176 & 732 \\
$24 \cdot 5$ & 308 & $0 \cdot 65$ & $75 \cdot 5$ & 3328 & 1818 & $\mathbf{6 8 6}$ \\
$15 \cdot 0$ & 400 & $0 \cdot 50$ & 60.0 & 2800 & 1600 & 700 \\
$8 \cdot 5$ & 572 & $0 \cdot 35$ & 48.6 & 2516 & $\mathbf{1 5 4 4}$ & 815 \\
3.5 & 1000 & $0 \cdot 20$ & 35.0 & $\mathbf{2 4 0 0}$ & 1700 & 1175 \\
\hline
\end{tabular}

$S_{1}$ and $m$. From this example, and from numerous other such tables, it emerges that there are minimal costs associated with the size of $P$ and the choice of $S_{1}$ and $m$. In general, one should prefer low $S_{1}$ and low $m$ (say 0.35 ) if second-stage trials are expensive, but high $S_{1}$ and high $m$ (say 0.65 ) if they are cheap. The reason clearly lies in the inverse relation (for fixed $b$ ) between $N$ and $(b+d)$, the two quantities which, with $P$, determine costs.

If $r$ is well known or can be confidently predicted, the best strategy is thus easily defined: choose $S_{1}$ and $m$ (within "reasonable" limits-see above) to minimise cost for the chosen outcome. Table 2 suggests a rule of thumb $(m=0.35$ for cheap trials, $m=0.65$ for dear ones) but any breeder contemplating strategy would do well to determine the best $m$ for his particular parameters. But the optima are not sharp and little will be lost by constraining $m$. Table 2 also illustrates the declining demands on numbers and total costs as the intensity of $S_{2}$ is relaxed; and the decline of total costs as $r$ increases.

I now consider the consequences of uncertainty as to the size of $r$. Obviously, if the optimal strategy is defined as above, but $r_{r} \neq r_{a}$, then two errors are possible: if $r_{r}>r_{a}$, there will be, for the

Table 2 Selected results to show the economic importance of the choice of $S_{1}$ and $m$ at three levels each of $r$ and $S_{2}$. The objective in each case is $b=10$ selections. The symbols $C_{40}$ and $C_{5}$ indicate costs at $P=40,5$, respectively

\begin{tabular}{|c|c|c|c|c|c|c|c|c|c|c|c|}
\hline \multirow[b]{2}{*}{$S_{2} \%$} & \multirow[b]{2}{*}{$m$} & \multirow[b]{2}{*}{$N$} & \multicolumn{3}{|c|}{$r=0.3$} & \multicolumn{3}{|c|}{$r=0 \cdot 5$} & \multicolumn{3}{|c|}{$r=0.7$} \\
\hline & & & $S_{1}$ & $C_{40}$ & $C_{5}$ & $S_{1}$ & $C_{40}$ & $c_{5}$ & $S_{1}$ & $C_{40}$ & $C_{5}$ \\
\hline \multirow[t]{2}{*}{5} & 0.35 & 572 & $16 \cdot 0$ & 4233 & 1030 & 8.5 & 2516 & 815 & $4 \cdot 0$ & 1488 & 686 \\
\hline & 0.65 & 308 & $40 \cdot 0$ & 5236 & 924 & 24.5 & 3328 & 686 & 12.5 & 1848 & 501 \\
\hline \multirow[t]{2}{*}{10} & 0.35 & 286 & 18.0 & 2345 & 543 & $11 \cdot 0$ & 1554 & $44 ?$ & 6.5 & 1030 & 379 \\
\hline & 0.65 & 154 & $43 \cdot 5$ & 2834 & 489 & $30 \cdot 0$ & 2002 & $38:$ & $17 \cdot 0$ & 1201 & 285 \\
\hline \multirow[t]{2}{*}{20} & 0.35 & 143 & 21.5 & 1373 & 297 & $15 \cdot 0$ & 1001 & 250 & $10 \cdot 0$ & 715 & 215 \\
\hline & 0.65 & 77 & 48.0 & 1555 & 262 & $36 \cdot 0$ & 1186 & 216 & $25 \cdot 0$ & 847 & 173 \\
\hline
\end{tabular}


calculated effort, more than the expected 10 (generally $b$ ) selections planned; but if $r_{r}<r_{a}$, there will be fewer selections of the desired standard, though for the same budgeted expenditure of effort. Both errors are diseconomies, the former because the excess represents effort that could have been spent elsewhere, the latter because a deficiency is an inconvenience to the programme and the selections will be expensive. Table 3 shows some results, with cheap and costly second-stage trials $(P=5,40)$ and appropriate $m$. The number $b$ may be nearly halved or doubled by a serious mischoice of $r_{a}$. Unit costs are not much affected and I think most breeders would regard a deficiency of $b$ as a more serious error than an excess. So, in uncertainty, a moderate pessimism as to $r_{a}$ would be reasonable.

So far we have considered solutions under a specified objective for $b$ but with $N$ and $C$ unconstrained. Constraints on either are conceivable, however. If $N$ were fixed at so low a level that only very small numbers of superior selections could be expected to be present, commonsense suggests that the breeder would do best to select weakly (set $S_{1}$ high) and aim at high $m$. As a measure of success we need an economic criterion. Several are possible and, fairly arbitrarily, I choose one which puts $F$ (a measure of the breeder's satisfaction) as proportional to $b$ and inversely proportional to unit cost $(C / b)$. Thus $F=1000$ $\left(b^{2} / C\right)$. Using this criterion, the commonsense view is indeed supported (table 4 ). If $N$ were fixed at a much higher level, so high that the breeder could always meet a chosen requirement as to the size of $b$, the problem disappears.

The case of fixed $C$ can be examined by adjusting $(b+d)$ and $N$ to produce the required $C$ (table 5 ). Since costs are fixed, optima are simply given by maximal $b$. Results are, perhaps surprisingly, indifferent as to $S_{2}$ (range 5-20) and $r$ (range $0 \cdot 3-0 \cdot 7)$. The rule is to choose low $m$ and $S_{1}$ when $P$ is high, high $m$ and $S_{1}$ when $P$ is low. Optima are weak, a wide range of $m$ and $S_{1}$ making little difference to the best $b$ (table 5). Nevertheless, optima are there and it is obviously possible to set $S_{1}$ grossly too low or too high (see sugarcane example below).

\section{EXAMPLES}

In sugarcane breeding, selection rates from the seedling stage to the first clonal stage vary rather widely from under 5 per cent to $10-15$ per cent.

Table 3 The results of uncertainty. Values of $b$ (objective $b=10$ ) for various values of $r_{a}$ and $r_{r}$.

\begin{tabular}{|c|c|c|c|c|c|c|c|c|}
\hline$S_{2}$ & $N$ & $P$ & $m$ & $r_{r}$ & $r_{a}=0.3$ & $r_{a}=0.5$ & $r_{a}=0.7$ & \\
\hline \multirow[t]{6}{*}{10} & 154 & 5 & 0.65 & $0 \cdot 3$ & & $10 \cdot 0$ & $7 \cdot 7$ & $5 \cdot 2$ \\
\hline & & & & $0 \cdot 5$ & & $12 \cdot 2$ & $10 \cdot 0$ & $7 \cdot 4$ \\
\hline & & & & $0 \cdot 7$ & & $14 \cdot 3$ & $12 \cdot 6$ & $10 \cdot 0$ \\
\hline & & & & & & $43 \cdot 5$ & $30 \cdot 0$ & $17 \cdot 0$ \\
\hline & & & & & $(b+d)$ & 67 & 46 & 26 \\
\hline & & & & & C & 489 & 385 & 285 \\
\hline \multirow[t]{6}{*}{20} & 77 & 5 & 0.65 & $0 \cdot 3$ & & $10 \cdot 0$ & $8 \cdot 2$ & $6 \cdot 2$ \\
\hline & & & & 0.5 & & $11 \cdot 7$ & $10 \cdot 0$ & $7 \cdot 9$ \\
\hline & & & & 0.7 & & $13 \cdot 7$ & $12 \cdot 0$ & $10 \cdot 0$ \\
\hline & & & & & & $48 \cdot 0$ & $36 \cdot 0$ & $25 \cdot 0$ \\
\hline & & & & & $(b+d)$ & 37 & 28 & 19 \\
\hline & & & & & C & 262 & 216 & 173 \\
\hline \multirow[t]{6}{*}{10} & 286 & 40 & 0.35 & 0.3 & & $10 \cdot 0$ & $6 \cdot 9$ & $4 \cdot 3$ \\
\hline & & & & 0.5 & & $14 \cdot 0$ & $10 \cdot 0$ & $6 \cdot 6$ \\
\hline & & & & $0 \cdot 7$ & & $18 \cdot 9$ & $14 \cdot 3$ & $10 \cdot 0$ \\
\hline & & & & & & $18 \cdot 0$ & $11 \cdot 0$ & $6 \cdot 5$ \\
\hline & & & & & $(b+d)$ & 51 & 31 & 19 \\
\hline & & & & & $C$ & 2345 & 1544 & 1030 \\
\hline \multirow[t]{6}{*}{20} & 143 & 40 & 0.35 & 0.3 & & $10 \cdot 0$ & $7 \cdot 4$ & $5 \cdot 4$ \\
\hline & & & & 0.5 & & $13 \cdot 1$ & $10 \cdot 0$ & $7 \cdot 4$ \\
\hline & & & & $0 \cdot 7$ & & $17 \cdot 1$ & $13 \cdot 4$ & $10 \cdot 0$ \\
\hline & & & & & & $21 \cdot 5$ & $15 \cdot 0$ & $10 \cdot 0$ \\
\hline & & & & & $(b+d)$ & 31 & 21 & 14 \\
\hline & & & & & $C$ & 1373 & 1001 & 715 \\
\hline
\end{tabular}


Table 4 The effect of constraining $N=100$, with $F=1000 b^{2} / C$ and $r=0 \cdot 5$. The best $F$ is always at high $S_{1}$ and $m=0.65$ or more

\begin{tabular}{|c|c|c|c|c|c|c|c|}
\hline \multirow[b]{2}{*}{$S_{2}$} & \multirow[b]{2}{*}{$m$} & \multirow[b]{2}{*}{$b$} & \multirow[b]{2}{*}{$S_{1}$} & \multicolumn{2}{|c|}{$P=40$} & \multicolumn{2}{|c|}{$P=10$} \\
\hline & & & & $C$ & $F$ & $C$ & $F$ \\
\hline \multirow[t]{4}{*}{5} & 0.35 & 1.75 & $8 \cdot 5$ & 440 & 7 & 185 & 17 \\
\hline & $0 \cdot 50$ & $2 \cdot 50$ & $15 \cdot 0$ & 700 & 9 & 250 & 25 \\
\hline & 0.65 & $3 \cdot 25$ & $24 \cdot 5$ & 1080 & 10 & 345 & 31 \\
\hline & 0.80 & $4 \cdot 00$ & $38 \cdot 5$ & 1640 & 10 & 485 & 33 \\
\hline \multirow[t]{4}{*}{10} & $0 \cdot 35$ & $3 \cdot 5$ & $11 \cdot 0$ & 540 & 23 & 210 & 58 \\
\hline & $0 \cdot 50$ & $5 \cdot 0$ & 19.0 & 860 & 29 & 290 & 86 \\
\hline & 0.65 & 6.5 & $30 \cdot 0$ & 1300 & 33 & 400 & 106 \\
\hline & $0 \cdot 80$ & $8 \cdot 0$ & $44 \cdot 5$ & 1880 & 34 & 545 & 117 \\
\hline \multirow[t]{4}{*}{20} & $0 \cdot 35$ & $7 \cdot 0$ & $15 \cdot 0$ & 700 & 70 & 250 & 196 \\
\hline & $0 \cdot 50$ & $10 \cdot 0$ & $24 \cdot 5$ & 1080 & 93 & 345 & 290 \\
\hline & 0.65 & $13 \cdot 0$ & $26 \cdot 0$ & 1540 & 110 & 460 & 367 \\
\hline & $0 \cdot 80$ & $16 \cdot 0$ & $53 \cdot 0$ & 2220 & 115 & 630 & 406 \\
\hline
\end{tabular}

Table 5 The optimum for fixed $C=500$ with $P=10, S_{2}=10$, $r=0 \cdot 5$. The optimum is at about $S_{1}=17, b=8 \cdot 7, F=151$

\begin{tabular}{rrlllr}
\hline \multicolumn{1}{l}{$S_{1}$} & $N$ & $m$ & $(b+d)$ & $b$ & \multicolumn{1}{c}{$F$} \\
\hline $5 \cdot 0$ & 333 & $0 \cdot 20$ & 16.7 & $6 \cdot 7$ & 90 \\
11.0 & 238 & $0 \cdot 35$ & $26 \cdot 2$ & $8 \cdot 3$ & 138 \\
$19 \cdot 0$ & 172 & 0.50 & 32.8 & $8 \cdot 6$ & 148 \\
$30 \cdot 0$ & 125 & 0.65 & 37.5 & $8 \cdot 1$ & 131 \\
$44 \cdot 5$ & 92 & 0.80 & 40.8 & $7 \cdot 3$ & 107 \\
\hline
\end{tabular}

Correlations between tests are moderate for the more repeatable characters such as Brix but low for yield and overall "worth" (which includes yield as a major component). Numbers of seedlings are traditionally large or very large. Several authors (e.g., Walker, 1960) have urged the adoption of as lax a first selection as possible in view of the low correlation and of recurrent experience of good clones that were not selected as seedlings (see also Simmonds, 1984). To see whether this view can be supported, I consider a typical population of $N=$ 10,000 , with $S_{1}=3$ per cent, $S_{2}=1$ per cent, $r=0 \cdot 2$ and $P=30$. It appears that $b=8,(b+d)=300$ and $C=19 ; m$ is only 0.08 which is so low (figure 1) as to make it very likely that the peak selections are removed. Exploring higher values of $S_{1}$ (with lower $N$ ), a broad optimum emerges at $S_{1}=10$ to 20 per cent with other parameters as follows; $m=$ $0 \cdot 22$ to $0 \cdot 28,(b+d)=364$ to $421, n=3600$ to 2100 and $C=14 \cdot 6$ to $14 \cdot 7$, all for a constant $b=8$. At a constant cost of $C=19, N$ and $(b+d)$, of course, rise a little and $b=10 \cdot 3$. In order to capture some of the peak selections, $S_{1}=18$ per cent with $m=$ 0.35 would be a reasonable (though not overgenerous) choice. If $r$ were taken to be as high as $0 \cdot 4, b$ becomes 19 , with $m=0 \cdot 19$, and the optimum
$S_{1}$ falls to about 7 per cent with $m=0.33$; the advantages of cost or of numbers of superior selections at constant cost are much less pronounced. However, $r$ as high as 0.4 is unlikely, so a prudent strategy would be to assume $r=0 \cdot 2$, confident that, if $r$ were, in fact, higher, the outcome would be more selections than expected and an acceptable $m$. The case for relatively weak first-stage selection is therefore clear. I note that James and Miller (1975), using quite different arguments, also made a case for weak first-stage selection in this crop.

I turn now to an example from rubber (Ong, 1981). Costs of preliminary trials (Small Scale Clone Trials, SSCT) and of second-stage trials (Large Scale Clone Trials, LSCT) are estimated as tree-years per clone, with $P=50$ to 200 , depending on the number of years of tapping accounted for. It is enough that $P$ is very large; the actual number is not critical. I consider only the 700 Series data since the two earlier Series trials (500 and 600) were less than satisfactorily designed. For various SSCT-LSCT yield comparisons, $r \approx 0.7$ (range 0.52 to 0.79$) ; 26$ clones were tested of which four were recommended but only one proved to be really good. Ong noted that two years of yield records in SSCT provided a good (and quick and cheap) prediction of long-term yield in LSCT. The rather high correlation suggests that selection from SSCT could well have been more stringent and so it turns out. With $S_{2}$ in the range $20-40$ per cent there are optima for $S_{1}$ in the range 35-60 per cent. For this material (and a small sample) $S_{2}$ less than 40 per cent would hardly be reasonable; with $S_{1}=$ $60, b=9 \cdot 1, m=0 \cdot 88$, the total cost of experimentation would have been roughly halved with little risk of loss of good selections. 
The 26 clones tested were the top end of a much larger body of clones tested SSCT. Clearly, it would be economical to increase the flow of clones into the relatively cheap SSCT and select fairly stringently for advance into LSCT. Since the correlation nursery-SSCT is known to be low (ca 0.3Tan, 1985; cf Simmonds, 1986), large seedling populations and fairly weak selection into SSCT are indicated (cf. the sugarcane example above).

\section{DISCUSSION}

The theory outlined in this paper relates to two stages only and is theoretically applicable only at the start of a programme before truncation disturbs normality. The effect of previous truncation will be to reduce $r$ but it will be for later studies to show how important this effect is. It may turn out that it is conservative in the sense of merely encouraging a moderate pessimism as to $r$ which, as we have seen, is reasonable. The effect of including families differing in means in a notional single population will be the opposite: it will tend to cause overdispersion and therefore spuriously to increase the estimate of $r$. This effect, too, will be for investigation; it may be that sub-populations with different means should be treated separately in the choice of $S_{1}$ and $S_{2}$.

The objective of the first stage of selection in a programme is not maximal progress as measured by the mean of the survivors. This would generally be achieved by intense selection (so long as $r>0$ ) and it would be estimated from the simple regression $b_{2.1}$. The objective, rather, is to select at stage 1 in such a way as to give assurance that a "reasonable" proportion of the best entries remain for subsequent selection. As we have seen, this means setting $m$ within working limits, reasonably (I think) taken to be 0.35 to 0.65 (but without being too rigorous in the matter). Certainly, too low $m$ removes the peak and too high $m$ is wasteful (figure 1). We should recall, incidentally, as noted above, that $S_{2}$ is not the actual selection rate in stage 2 but a notional fraction intended only to include some of the very best. $S_{2}$ may be 1-5 per cent but the overall rate of success after repeated cycles may well be (e.g., in sugarcane) of the order $10^{-5}$ to $10^{-6}$ (cf. Simmonds, 1984). Several authors have pointed out that weak early selection may be a cost-effective way of preserving good entries for later identification (e.g., Briggs and Shebeski, 1970; Geadelmann and Frey, 1974; James and Miller, 1975).
Numerous replications of a standard variety in stage 1 could be very helpful in defining a wellestimated cut-off point and hence in setting possible values for $S_{1}$. The consequences for stage 2 could thus be roughly calculated and the breeder would $\ddot{b e}$ in a position to adjust $S_{1}$ to aim at a chosen value of $b$ or to maximise $b$ within cost constraints. One useful conclusion from such referral to a standard could, of course, be that $S_{1}$ should be set at zero and resources transferred to more promising material. In the possible presence of strong genotype $\times$ year interactions, the cautious breeder might reflect that to base decision as to $S_{1}$ upon an arbitrary standard in one year could disqualify some good genotypes more or less randomly. In that case, he could reasonably explore the consequences for stage 2 and for costs of relaxing $S_{1}$. Since most stage-to-stage correlations are based on experiments confounded with years, $\mathrm{GE}$ effects presumably contribute, maybe quite substantially, to weakening the $r$.

One consequence for the breeder of making calculations of the kind described here is to concentrate attention on the value of $P$, which might be adjusted by modification of either the first or the second trials. It might be, for example, that $r$ could be improved by enhancing the first stage trial at the expense of the second without detriment to total cost. Certainly, no review of a system should ignore the possibility of adjusting $P$.

The methods described have somewhat wider applications in plant breeding than two-stage selection. Thus, in selecting for a quality character, the breeder might have data from cheap, inaccurate measurements known to be correlated with expensive but accurate ones. It would then be feasible to calculate an optimal procedure whereby only a defined fraction of the population need be subjected to the expensive test (example in Simmonds, 1983).

Finney (1958) gave a general treatment of multi-stage selection, treating yield as a continuous variate, with all the statistical complications implied by successive truncation. His leading conclusions were that: (1) roughly constant total resources should be deployed in each cycle of trials, declining numbers being balanced by increasing replication; (2) having taken account of the costs of trials, broad optima were always apparent; (3) it would sometimes be reasonable to discard material at random in order to concentrate resources on the better testing of fewer entries. Points (2) and (3) emerge also in the present study: there is always a broad (not sharply defined) optimum and it would sometimes be best to reduce 
$N$ in favour of enhanced $(b+d)$. On point $(1)$ there is only partial agreement. I find that, at the optimum, generally, $P(b+d)>N$ but that $P(b+d) \leqslant N$ when $r$ is high and $S_{2}$ low. So an "equal resources" rule is sometimes but not always true and the relation is not such as to provide a guide to strategy.

Acknowlegements I am grateful to $\mathrm{Mr}$ D. I. T. Walker for discussion of sugarcane breeding problems and to Dr F. J. W. England for helpful criticism. Especial thanks are due to $\mathrm{Dr}$ H. D. Patterson for calculating the contents of the Appendix without which this study would have been impossible.

\section{REFERENCES}

BRIGGS, K. G. AND SHEBESKI, L. M. (1970). Visual selection for yielding ability of $F_{3}$ lines in a hard red spring wheat breeding program. Crop Sci., 10, 400-402.

DIGBY, P. G. N. (1983). Approximating the tetrachoric correlation coefficient. Biometrics. 39, 753-757.

ENGLAND, F. J. W. (1967). Non-sward densities for the assessment of yield in Italian rye-grass 1. J. Agric. Sci. Camb., $68,235-241$.
FINNEY, D, J. (1958). Plant selection for yield improvement. Euphytica, 7, 83-106.

GEADELMANN, J. L. AND FREY, K. J. (1975). Direct and indirect mass selection for grain yield in bulk oat populations. Crop Sci., 15, 490-494.

GILBERT, N. E. (1961). Correlations in plant breeding. Euphytica, 10, 205-208.

JAMES, N. I. AND MILleR, J. D. (1975). Selection in six crops of sugarcane. 11. Crop Sci., 15, 37-40.

KEULS, M. AND SIEBEN, J. W. (1955). Two statistical problems in plant selection. Euphytica, 4, 34-44.

ONG, s. H. (1981). Correlations between yield, girth and bark thickness of RRIM clone trials. J. Rubb. Res. Inst. Malaysia, $29,1-14$.

SIMMONDS, N. W. (1983). Contingency and correlation: some applications to sugarcane breeding. ISSCT Sugar Cane Brdrs Newsl., 4, 20-25.

SIMMONDS, N. W. (1984). A note on the strategy of breeding clonal crops. Heredity, 53, 397-401.

SIMMONDS, N. W. (1986). Rubber breeding. In Webster, C. C. and Baulkwill, J. (eds) Rubber, Longman, London, in the press.

TAN, H. (1985). Current status of Hevea breeding. In Campbell, A. I., Abbot, A. J. and Atkin, R. K. (eds). Improvement of Vegetatively Propagated Crops. Academic, London, in the press. (8th Long Ashton Symposium, 1982).

WALKER, D. I. T. (1960). Useful information from variety trials. Proc. Brit. W. Ind. Sugar Tech., 1960. 15-27. 
Appendix 1 Tabulation of $b\left(\times 10^{3}\right)$ for various $S_{1}$ and $S_{2}$ (see Figure 1 and text). In each cell are given nine entries for $b$ corresponding with $r=0 \cdot 1,0 \cdot 2, \ldots, 0 \cdot 9$. Example; $S_{1}=30$ per cent, $S_{2}=10$ per cent, $r=0 \cdot 5$, then $b=65$ and $a=35, c=665, d=235, m=0.65$ or 65 per cent.

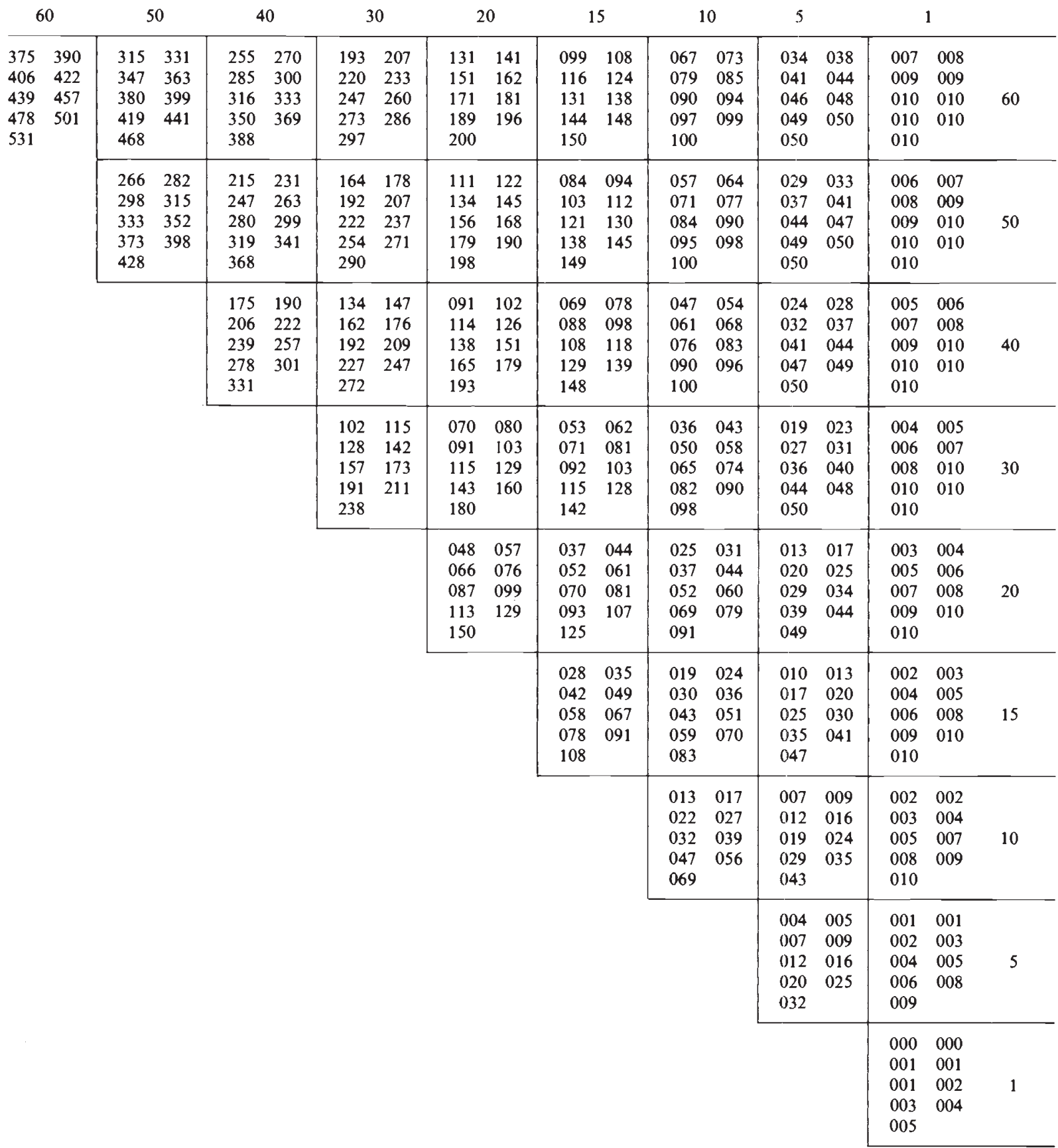

